\title{
Superdiffusion in a Honeycomb Billiard
}

\author{
Michael Schmiedeberg and Holger Stark \\ Universität Konstanz, Fachbereich Physik, D-78457 Konstanz, Germany
}

\begin{abstract}
We investigate particle transport in the honeycomb billiard that consists of connected channels placed on the edges of a honeycomb structure. The spreading of particles is superdiffusive due to the existence of ballistic trajectories which we term perfect paths. Simulations give a time exponent of 1.72 for the mean square displacement and a starlike, i.e., anisotropic particle distribution. We present an analytical treatment based on the formalism of continuous-time random walks and explain both the time exponent and the anisotropic distribution. In billiards with randomly distributed channels, conventional diffusion is always observed in the long-time limit, although for small disorder transient superdiffusional behavior exists. Our simulation results are again supported by an analytical analysis.
\end{abstract}

PACS numbers: 05.40.Fb,05.60.Cd

\section{INTRODUCTION}

Billiard systems, i.e., point particles moving freely in areas bounded by closed curves from which they reflect specularly, are a paradigm of classical mechanics illustrating the difference between regular and chaotic motion 1, 2]. Moreover, they are helpful to explore the relation between classical and quantum mechanics [3], where interference of waves becomes important as also observed in optical resonators [4] or by chaotic scattering in optical billiards 5].

In this paper we investigate a special example of the so-called infinite domain or extended billiard, where particles' motion is unrestricted. The most famous example invented by Lorentz [6] to model electrons in a metal is the Lorentz gas, where particles reflect specularly from randomly distributed spherical scatterers. A periodic version, the so-called Sinai billiard 7], is illustrated in Fig. 19). For special paths, the particles possess an infinite horizon, i.e., they move straight without being scattered [see Fig. [1])]. These paths are responsible for the observation that a collection of particles with arbitrary initial direction experience superdiffusion, i.e., in the Sinai billiard their mean-square displacement grows as $t \ln t$, where $t$ denotes time [8]. If sufficiently large scatterers are placed on a hexagonal lattice 9] [see Fig. 10)] or if small scatterers fill the interstitial space of the Sinai billiard in Fig. 11) [10], particles always have a finite horizon and their motion is purely diffusive.

Recently, transport properties in one-dimensional extended billiards have been studied [10, 11, also with special emphasis on heat conduction [12, 13]. One example, a periodic arrangement of channels (see Ref. [11]), is pictured in Fig. 15). Although all particles in such a channel billiard have a finite horizon, there exist paths

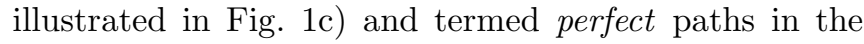
following, where the particles always move ballistically in one direction and therefore cause superdiffusion.

In this paper, we study a two-dimensional extended billiard, where channels are placed on the edges of the honeycomb structure, and denote it honeycomb billiard a)

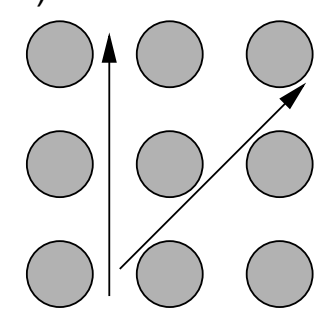

b)

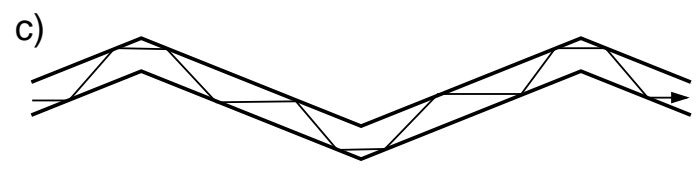

FIG. 1: a) Ballistic paths in a Sinai billiard, where particles experience an infinite horizon, give rise to superdiffusion. b) If the scatterers on hexagonal lattice points are sufficiently large, the particle trajectories always have a finite horizon and the spreading of particles is diffusive. c) A one-dimensional channel billiard with a perfect path giving rise to superdiffusion.

(see Fig. 2). The spreading of particles in such a billiard is also superdiffusive due to the existence of numerous ballistic or perfect paths examples of which are illustrated in Fig. 2 Note that path (3) is equivalent to the one in the one-dimensional billiard of Fig. 1). Our system is an example where particles perform a Lévy walk [16, 17, 18]. Very long effective steps along almost perfect paths lead to superdiffusion. We study it with the help of computer simulations and motivate the time exponent for the particles' mean square displacement within the velocity model [19] of continuous-time random walks 17, 18]. We furthermore look at random distortions of the honeycomb billiard and show that for small distortions a transient superdiffusive regime exists whereas for large times and also for large distortions the spreading of the particles is always diffusive. Our numerical results are again supported by an analytical analysis.

Originally, our work was motivated by the observation of photon channelling in foams [14, 15]. Light in foams is reflected at the liquid-gas interface of the thin films 


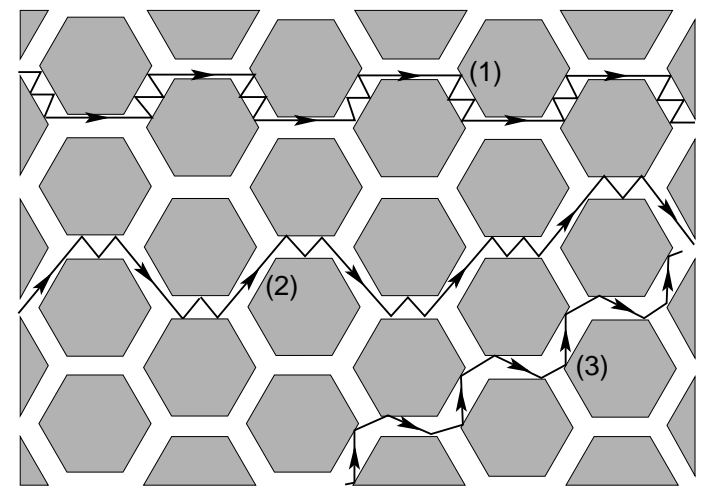

FIG. 2: Honeycomb billiard with three examples of perfect paths.

which ultimately leads to a diffusive transport of photons through the system. Experiments and theoretical considerations show that the photons have a higher probability to move in the liquid phase of the films, a phenomena that was then termed photon channelling. The channel billiards studied here are an extreme case where the photons always move in the liquid phase.

Finally, we add a note concerning the classification of our billiard system. Arnol'd's famous theorem states that the phase space of integrable systems in classical mechanics is a torus [20]. On the other hand, the Lorentz gas is chaotic and therefore nonintegrable [3]. Besides quasi-integrable systems [2], Richens and Berry identify pseudointegrable systems with chaotic properties whose phase space is a multi-handled sphere instead of a torus 21]. As an example, they investigate a system similar to the honeycomb billiard but with the hexagons replaced by squares and show that the corresponding phase space is a five-handled sphere [21]. Performing an analogous investigation for the honeycomb billiard, we find a tenhandled sphere as phase space [22].

In the following, we first introduce details of our billiard system and the method of simulations. In Section III we report our numerical results, discuss analytical approaches in Section IV and then end with conclusions. Appendix $\mathrm{A}$ contains details of the Lévy-walk model for the honeycomb billiard.

\section{MODEL SYSTEM AND METHOD OF SIMULATIONS}

The objective of this paper is to study the dynamics of particles in two-dimensional channel billiards. The construction of the regular honeycomb billiard, as illustrated in Fig. 2 is obvious. However, we also want to investigate random channel billiards. To create them, we employ Voronoi tessellations of the plane [24, 25]. They are generated from a distribution of seed points in a simulation box, for which Voronoi polygons are constructed in complete analogy to the Wigner-Seitz cells of period- ically arranged lattice sites. For example, a triangular lattice of seed points gives the regular honeycomb structure whose edges we choose to have the length $l_{0}$. In the following all lengths are given in units of $l_{0}$. Then we systematically introduce disorder by shifting the seed points along a randomly chosen displacement vector whose magnitude is equally distributed in the intervall $[0, \delta r]$. All of our Voronoi tessellations are produced by the software Triangle [26]; examples are presented, e.g., in Ref. [27. Typically, they contain approximately 15000 cells which corresponds to a quadratic simulation box with edge length $200 l_{0}$. This simulation box is extended in all spatial directions by periodic boundary conditions.

Now, we arrive at a random channel billiard by placing a channel of width $d$ on each edge of the Voronoi tessellation. Only modest disorder quantified by $\delta r \leq 0.3$ is investigated so that all cells still have six edges. This avoids the situation that four instead of three channels meet when we construct the billiard system, which simplifies the determination of the particle path. Particles perform a ballistic motion with a constant velocity $c$ inside the channels; when they hit the boundary they are reflected specularly. In the following, we use the time scale $l_{0 / c}$ to rescale time.

Typically, we launch 10000 particles at one vertex of the underlying Voronoi tesselation in an angular range of $60^{\circ}$ and let them run during a time $t=10^{5}$. At several times, we calculate the mean square displacement $\left\langle\mathbf{r}^{2}\right\rangle$, where $\mathbf{r}$ denotes the position vector of the particles in the particle cloud, and plot it as a function of $t$. When applicable, diffusion constants in units of $l_{0} c$ are then determined from a fit to $\left\langle\mathbf{r}^{2}\right\rangle=4 D t$.

\section{SUPERDIFFUSION: RESULTS FROM SIMULATIONS}

In this section we present our numerical results for the exact honeycomb billiard and the random channel billiard. Figure 3, where we plot the mean-square displacement as a function of time for different $\delta r$, summarizes our main results. In the exact honeycomb billiard $(\delta r=0$, plus symbols), the particles exhibit superdiffusion, i.e., in $\left\langle\mathbf{r}^{2}\right\rangle \propto t^{\nu}$ the time exponent is larger than one and assumes the value $\nu=1.72 \pm 0.02$. Within the numerical error, the exponent is independent of the channel width $d$ as long as $d$ is small enough so that the particle's horizon is finite. Nevertheless, even for a finite horizon, we find ballistic trajectories, termed perfect paths in the following, in the sense that the particles move, on average, in one direction although they experience numerous reflections in the channels. Examples of such trajectories are illustrated in Fig. 2 path (3) is equivalent to the "propagating periodic orbit" in the work of Sanders and Larralde [see Fig. 10) and Ref. [10]]. In the framework of Lévy walks, they can be considered as steps of infinite length and therefore are responsible for the superdiffusive behavior. We will investigate them in more detail 


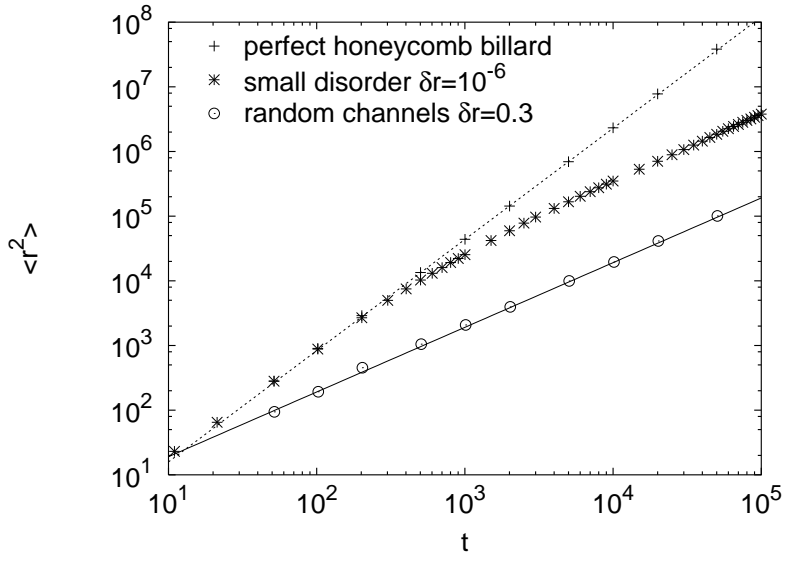

FIG. 3: Mean-square displacement as a function of time for the perfect honeycomb billiard, for small and large disorder. The symbols are results from simulations, the dashed line is a fit, and the full line is based on an analytic result for the diffusion constant [see Eq. (24)]. The channel width is $d=0.1$. a)

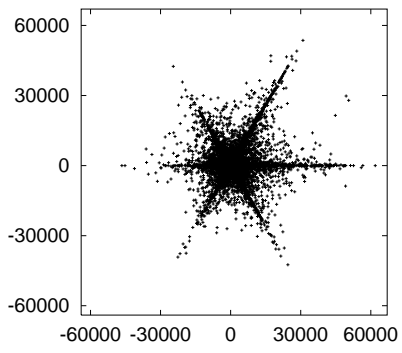

b)

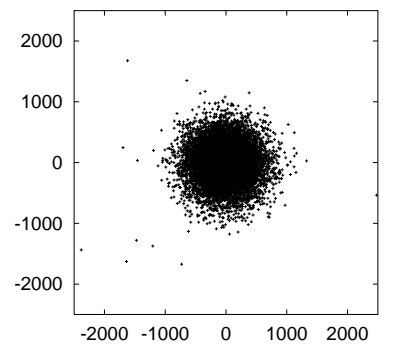

FIG. 4: Particle distribution for $t=10^{5}$ for (a) the honeycomb billiard and (b) the random channel billiard with $\delta r=0.3$. The channel width is $d=0.1$.

in section IV For small disorder $\left(\delta r=10^{-6}\right.$, star symbols), the mean-square displacement exhibits a transient superdiffusive regime for small times with the same exponent $\nu=1.72$ as in the regular case and then enters conventional diffusion $(\nu=1)$ for large times. Finally, for large disorder $(\delta r=0.3$, circle symbols), the motion is purely diffusive.

Figure 4a) shows clearly that the superdiffusive motion in the honeycomb billiard is associated with a nonisotropic probability distribution $P(\mathbf{r}, t)$ of the particles. The spikes in $P(\mathbf{r}, t)$, plotted for $t=10^{5}$, suggest that the long effective steps, responsible for superdiffusion, occur along the six equivalent directions of the channels. Within the theory of continuous-time random walks, we can show that such a spiky shape of the distribution has to appear. However, in the regime of conventional diffusion $(\delta r=0.3)$, the distribution $P(\mathbf{r}, t)$ assumes the expected isotropic shape of the Gaussian distribution, as illustrated in Fig. 4b).

We investigated if the probability distribution $P(\mathbf{r}, t)$

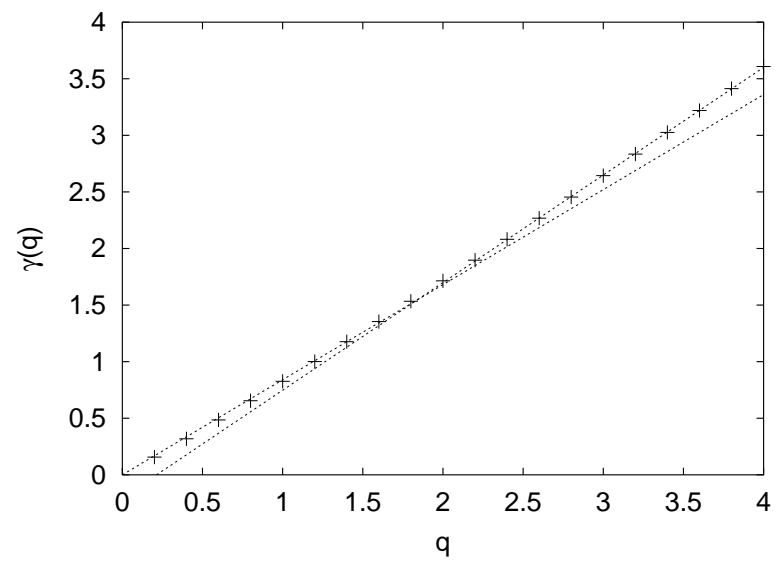

FIG. 5: Time exponent $\gamma(q)$ for the $q$ th moment of the particle distribution function plotted versus $q$. The symbols are results from simulations for the exact honeycomb billiard with channel width $d=1$. The fitted dashed lines have slopes $1.68 / 2$ and $1.72 / 2$.

obeys the scaling law:

$$
P(\mathbf{r}, t)=\frac{1}{t^{\nu}} P\left(\frac{\mathbf{r}}{t^{\nu / 2}}, 1\right)
$$

If it is valid, the moments of $P(\mathbf{r}, t)$ fulfill

$$
\left\langle|\mathbf{r}(t)|^{q}\right\rangle=t^{\gamma(q)}\left\langle|\mathbf{r}(1)|^{q}\right\rangle \text { with } \gamma(q)=\nu q / 2,
$$

as one can show in a straightforward manner. We determined the exponents $\gamma(q)$ from a double-logarithmic plot of $\left\langle|\mathbf{r}(t)|^{q}\right\rangle /\left\langle|\mathbf{r}(1)|^{q}\right\rangle$ versus time $t$. Figure 5 plots $\gamma(q)$ as a function of $q$ for the honeycomb billiard for a channel width $d=1$. Since the single points follow a nearly straight line, the scaling law of Eq. (11) is roughly fulfilled. A closer inspection, however, reveals that the regions for $q<2$ and $q>2$ are better fitted by different slopes $\nu=1.68$ and $\nu=1.9$, respectively. So the exponent $\nu=1.72$ determined from the mean-square displacement lies between these two values. Interestingly, such a small difference of the slopes was also found by Sanders and Larralde in Ref. [10] for their "parallel zigzag billiard" with the kink at $q=3$. On the other hand, in the infinite horizon billiards with $\left\langle\mathbf{r}^{2}\right\rangle \propto t \ln t$ studied by Armstead et al. [23], the same analysis also reveals a kink at $q=2$ but with a larger difference of the slopes $\nu=0.5$ for $q<2$ and $\nu=1$ for $q>2$, respectively. Finally, we note that for random channel billiards $\gamma(q)=q / 2$ confirming the expected Gaussian distribution.

In Fig. 6 we plot $\left\langle\mathbf{r}^{2}\right\rangle$ versus time for different strengths of disorder. At small times, there is always superdiffusional behavior with the same time exponent. Both the crossover time to conventional diffusion and the diffusion constant $D$ increase with decreasing $\delta r$. Especially, we find that $D$ diverges for $\delta r \rightarrow 0$. 


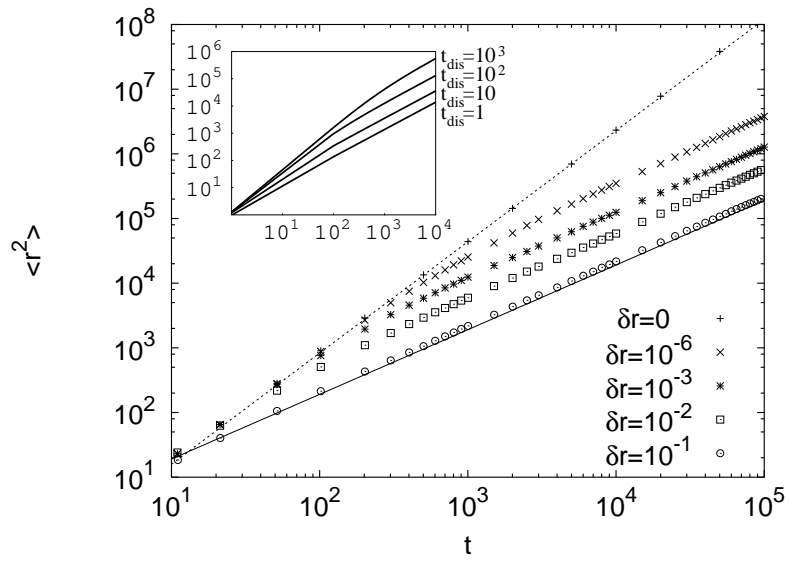

FIG. 6: Mean-square displacement as a function of time $t$ for different strengths $\delta r$ of disorder illustrating the transient regime of superdiffusion. The full and dashed lines indicate the limiting cases of conventional diffusion and pure superdiffusion. The symbols are results from simulations, the channel width $d$ is 0.1 . The inset shows the mean-square displacement calculated with the step-time distribution of Eq. (18) for different $t_{d i s}$.

\section{SUPERDIFFUSION: AN ANALYTIC APPROACH}

\section{A. Exact Honeycomb Billiard}

In Fig. 17 we plot randomly chosen particle trajectories from our simulations. They demonstrate that particles move, on average, in one direction for a very long time before they change their course. Moreover, long steps along one of the six possible channel directions are in the majority. These long steps occur due to the existence of ballistic trajectories or perfect paths, as we call them, where particles follow forever a certain direction. Examples were already introduced in Fig. 2] The main contribution to superdiffusion comes from paths that are close to perfect, i e., paths whose initial conditions differ slightly from a perfect one. Almost perfect and perfect trajectories then take the same channels for a long time until the difference between them is so large that they enter different channels. Therefore, an almost perfect path follows the direction of a ballistic trajectory for a long time before changing its direction. In the following, we consider the straight sections of an almost perfect path as an effective step in a Lévy walk and use the formalism of a continous-time random walk to determine the meansquare displacement and the particle distribution function on the basis of a distribution for these effective steps [19. Since in our case, the long steps are restricted to special directions, the exponent in the mean-square displacement is equivalent to the one found in Ref. 17] for pure one-dimensional rather than two-dimensional systems, as we will demonstrate explicitely below.

In a periodic Lorentz gas with infinite horizons the situ-

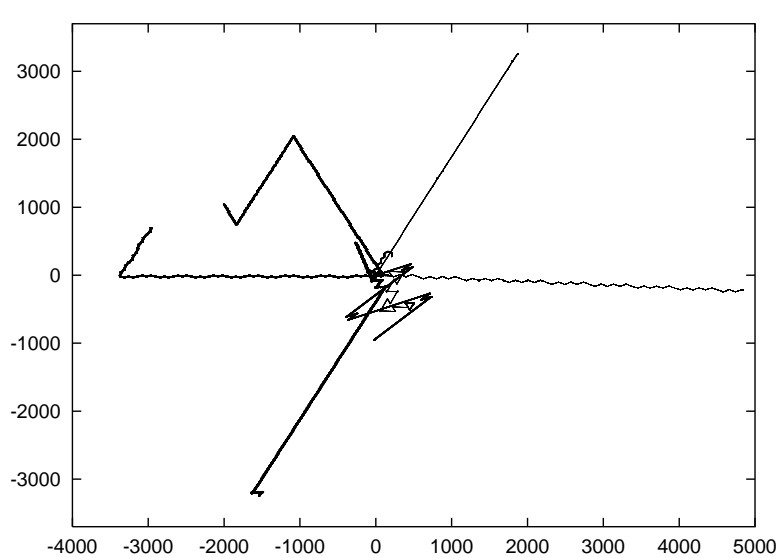

FIG. 7: Simulated paths of particles started with random conditions in a perfect honeycomb lattice. Lengths are given in units of the edge length $l_{0}$ of a hexagon. It is obvious that some particles follow straight paths along the six lattice axis for very long times before they change direction.

ation is similar. The main contribution to superdiffusion is due to paths close to the straight ballistic trajectories depicted in Fig. 17). An effective step in the Lorentz gas ends when a particle whose initial conditions differ from the ones of a ballistic path hits a scatterer. For both the Lorentz gas and the hexagonal billard, let us denote by $\epsilon$ the difference in the starting angle of a perfect and an almost perfect path. Particles starting at the same point will accumulate a difference $\delta x$ in position that in leading order grows as $t \epsilon$, where $t$ is the travel time of the particles. An effective step ends if $\delta x$ exceeds some treshold $\delta x_{\max }$ proportional to the distance of scatterers in the Lorentz gas or to the channel width $d$ in the honeycomb billard. In both systems, the duration $t$ of an effective step is therefore proportional to $1 / \epsilon$ for sufficiently small $\epsilon$. For the Lorentz gas this relation follows from the work in Ref. [8]; for the honeycomb billiard we explicitely justify it for a special class of perfect paths in Appendix A

Let $p(\epsilon) d \epsilon$ be the probability of finding a particle close to a perfect path with a starting angle in the intervall $[\alpha+\epsilon, \alpha+\epsilon+d \epsilon]$, where $\alpha$ is the starting angle of the perfect path. With $t \propto 1 / \epsilon$, one finds the distribution function $\lambda(t)$ of the effective step times:

$$
\lambda(t) d t=p(\epsilon) d \epsilon \propto \frac{p(1 / t)}{t^{2}} d t .
$$

In a Lorentz gas $p(\epsilon) \propto \sin \epsilon$, i. e., the velocity component $\cos \epsilon$ perpendicular to the direction of the infinite horizon is equally distributed [8]. Therefore, $\lambda(t) \propto 1 / t^{3}$ in the long-time limit that leads to a mean-square displacement $\left\langle\mathbf{r}^{2}\right\rangle$ proportional to $t \ln t[8,19]$ characterizing a marginally anomalous diffusion.

In our simulations of the particle transport in the honeycomb billard, we choose all starting angles with the same probability. A constant angular distribution $p(\epsilon)$ 
leads to $\lambda(t) \propto 1 / t^{2}$ and therefore $\left\langle\mathbf{r}^{2}\right\rangle \propto t^{2} / \ln t[19$. However, in the simulations we find $\left\langle\mathbf{r}^{2}\right\rangle \propto t^{1.72}$. We suspect that during the evolution of the photon cloud the angles close to perfect paths are no longer equally distributed, as also observed in the periodic Lorentz gas. In the following, we therefore consider an angular distribution $p(\epsilon) \propto \epsilon^{\beta}$ with an exponent $\beta$ between 0 and 1 in Eq. (3). To obtain a normalizable step-time distribution $\lambda(t)$, we use a small cutoff at a time $\tau$ and arrive at

$$
\lambda(t) \propto \frac{1}{t^{2+\beta}} \theta(t-\tau),
$$

where the step function $\theta(t-\tau)$ is one for $t>\tau$ and zero for $t<\tau$. In the formalism of continuous-time random walks, the important quantity is the probability $\psi(\mathbf{r}, t)$ that the particle performs a step along $\mathbf{r}$ in time $t$. It is written as $\psi(\mathbf{r}, t)=p(\mathbf{r} \mid t) \lambda(t)$, where $p(\mathbf{r} \mid t)$ denotes the conditional probability to move along $\mathbf{r}$ in time $t$. As suggested by Fig. 7 we assume that the particles move with an effective velocity $v$ along the six possible channel directions given by the unit vectors $\mathbf{e}_{j}=(\cos \pi j / 3, \sin \pi j / 3)$. So the conditional probability becomes

$$
p(\mathbf{r} \mid t)=\frac{1}{6} \sum_{j=1}^{6} \delta\left(\mathbf{r}-v t \mathbf{e}_{j}\right)
$$

We now calculate the particle distribution function $P(\mathbf{r}, t)$ and the mean-square displacement using the "velocity model" for Lévy-Walks introduced in [19]. Within the formalism of continuous-time random walks, the particle distribution function, i. e., the probability to find a particle at location $\mathbf{r}$ and time $t$, is given by the integral equation:

$$
\begin{aligned}
P(\mathbf{r}, t)= & \int d^{2} r^{\prime} \int_{0}^{t} d t^{\prime} P\left(\mathbf{r}^{\prime}, t^{\prime}\right) \psi\left(\mathbf{r}-\mathbf{r}^{\prime}, t-t^{\prime}\right) \\
& +R(\mathbf{r}, t),
\end{aligned}
$$

where $R(\mathbf{r}, t)$ is the probability to reach or pass the point $\mathbf{r}$ at time $t$ within one step:

$$
R(\mathbf{r}, t)=p(\mathbf{r} \mid t) \int_{t}^{\infty} d t^{\prime} \lambda\left(t^{\prime}\right) .
$$

Performing a Fourier transformation in space and a Laplace transformation in time, Eq. (6) can be solved:

$$
\bar{P}(\mathbf{k}, u)=\frac{\bar{R}(\mathbf{k}, u)}{1-\bar{\psi}(\mathbf{k}, u)}
$$

Here the bar indicates a function in Fourier-Laplace space. Using $\psi(\mathbf{r}, t)=p(\mathbf{r} \mid t) \lambda(t)$ together with the respective definitions (15) and (7) for $p(\mathbf{r} \mid t)$ and $R(\mathbf{r}, t)$, we find:

$$
\bar{P}(\mathbf{k}, u)=\frac{\sum_{j=1}^{6}\left[1-\bar{\lambda}\left(u-i v \mathbf{k} \cdot \mathbf{e}_{j}\right)\right] /\left[u-i v \mathbf{k} \cdot \mathbf{e}_{j}\right]}{\sum_{j=1}^{6}\left[1-\bar{\lambda}\left(u-i v \mathbf{k} \cdot \mathbf{e}_{j}\right)\right]}
$$

The Laplace transform of the step-time distribution (4) can be expanded for small $u$;

$$
\bar{\lambda}(u) \approx 1+a u^{1+\beta}-b u,
$$

where $a=\Gamma(-1-\beta)$ and $b=(1+\beta) \tau / \beta$ are positiv constants and $\Gamma(x)$ denotes the Gamma function. Note that the normalization $\int_{0}^{\infty} \lambda(t) d t=1$ is fulfilled by $\bar{\lambda}(0)=1$. Then, for small $u$ and $k$, Eq. (9) becomes

$$
\bar{P}(\mathbf{k}, u)=\frac{\sum_{j=1}^{6}\left[-a\left(u-i v \mathbf{k} \cdot \mathbf{e}_{j}\right)^{\beta}+b\right]}{\sum_{j=1}^{6}\left[-a\left(u-i v \mathbf{k} \cdot \mathbf{e}_{j}\right)^{1+\beta}+b u\right]} .
$$

In the denominator, the linear term in $\mathbf{k}$ has vanished because of $\sum_{j=0}^{6} \mathbf{k} \cdot \mathbf{e}_{j}=0$.

The Laplace transform of the mean-square displacement can be calculated with the help of $\bar{P}(\mathbf{k}, u)$ :

$$
\left\langle\overline{\mathbf{r}^{2}}(u)\right\rangle=-\left.\nabla_{\mathbf{k}}^{2} \bar{P}(\mathbf{k}, u)\right|_{\mathbf{k}=\mathbf{0}} .
$$

We are therefore taking a closer look at Eq. (9) in the limit $k / u \rightarrow 0$. We expand $\left(u-i v \mathbf{k} \cdot \mathbf{e}_{j}\right)^{\beta}$ and $\left(u-i v \mathbf{k} \cdot \mathbf{e}_{j}\right)^{1+\beta}$ in terms of $k / u$ and finally obtain

$$
\bar{P}(\mathbf{k}, u) \approx \frac{1}{u}-\frac{1}{2} \frac{a}{b} \beta v^{2} k^{2} u^{\beta-3} .
$$

Using this approximate form in Eq. (12) and calculating the inverse Laplace transform, we find the mean-square displacement in the long-time limit

$$
\left\langle\mathbf{r}^{2}(t)\right\rangle \propto t^{2-\beta} .
$$

In the region $0<\beta<1$, to which our calculations apply, the time exponent $\nu=2-\beta$ varies between one and two so that we are in the superdiffusive but subballistic regime. As already mentioned above, the relation between the exponents in the step-time distribution $\lambda(t)$ and the mean-square displacement is the same as the one found in Refs. 17, 18, 19] for one-dimensional systems. The exponent $\nu=1.72$, which we observe in our simulations, is achieved with an exponent $\beta=0.28$ in the angular distribution $p(\epsilon) \propto \epsilon^{\beta}$. Our numerical results can therefore be explained with the assumption that close to perfect paths the density of possible particle paths sharply drops to zero.

Fig. 8 shows $\bar{P}(\mathbf{k}, u)$ as given in Eq. (9) for $u=10^{-3}$, $v=1$ and $\beta=0.28$. One clearly sees a six-fold symmetry that through the inverse Fourier-Laplace transformation is also visible in $P(\mathbf{r}, t)$. To analyze this star-like distribution pattern further, we investigate $\bar{P}(\mathbf{k}, u)$ in the limit $u / k \rightarrow 0$. Expanding Eq. (9) in $u / k$ and employing polar coordinates, $\mathbf{k}=\left(k \cos \varphi_{k}, k \sin \varphi_{k}\right)$, we obtain

$$
\bar{P}(\mathbf{k}, u) \approx \frac{1}{u}+\frac{a}{b} v^{1+\beta} \frac{k^{1+\beta}}{u^{2}} \Phi\left(\varphi_{k}\right),
$$

where $\Phi\left(\varphi_{k}\right)$ is a function that only depends on the angular variable:

$$
\Phi\left(\varphi_{k}\right)=\frac{1}{6} \sum_{j=1}^{6}\left[i \cos \left(\varphi_{k}-\frac{\pi}{3} j\right)\right]^{1+\beta} .
$$




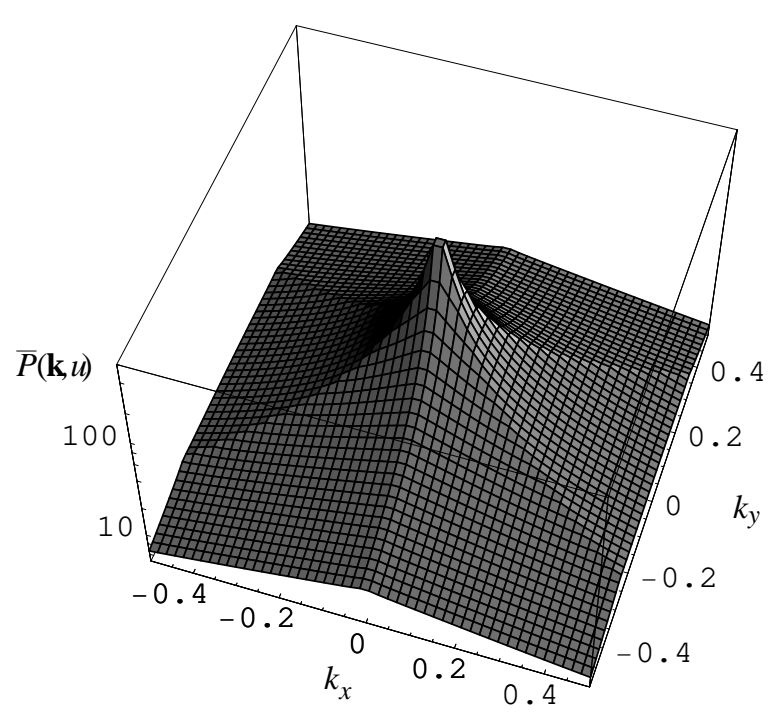

FIG. 8: Fourier-Laplace transform of the particle distribution function, $\bar{P}(\mathbf{k}, u)$, as given by Eq. (9) for $u=10^{-3}, v=1$ and $\beta=0.28$.

Due to the six-fold symmery, $\Phi\left(\varphi_{k}\right)$ is a real function and can also be written as

$$
\Phi\left(\varphi_{k}\right)=-\frac{1}{3} \sin \left(\beta \frac{\pi}{2}\right) \sum_{l=1}^{3}\left|\cos \left(\varphi_{k}-\frac{\pi}{3} l\right)\right|^{1+\beta} .
$$

We plot $\Phi\left(\varphi_{k}\right)$ in Fig. 9 for $\beta=0.28$. It has minima at $\phi_{k}=j \pi / 3(j=1 \ldots 6)$, indicating that along these directions the width of $\bar{P}(\mathbf{k}, u)$ is smaller compared to other directions, as is also visible in Fig. 8 Therefore, in real space the width of the particle distribution will be largest along the corresponding six channel directions. This explains the non-isotropic distribution pattern we find in our simulations [see Fig. [4 $)$ ]). So the star-like structure is indeed a result of the fact that effective long steps only occur along special directions.

\section{B. Random Channel Billiard}

In a disordered channel system, perfect paths do not exist. However, for small disorder, there are particle trajectories that pass the same channels as a perfect path until they ultimately take another route. This behavior is similar to the almost perfect paths in the honeycomb billiard. Small disorder therefore cuts off very long steps. To take this effect into account, we introduce an exponential factor in the step-time distribution:

$$
\lambda_{d i s}(t) \propto e^{-t / t_{d i s}} \lambda(t) \propto \frac{e^{-t / t_{d i s}}}{t^{2+\beta}} \theta(t-\tau),
$$

where $t_{d i s}$ is a time that decreases with increasing disorder $\delta r$. Paths with effective step times beyond $t_{d i s}$ are

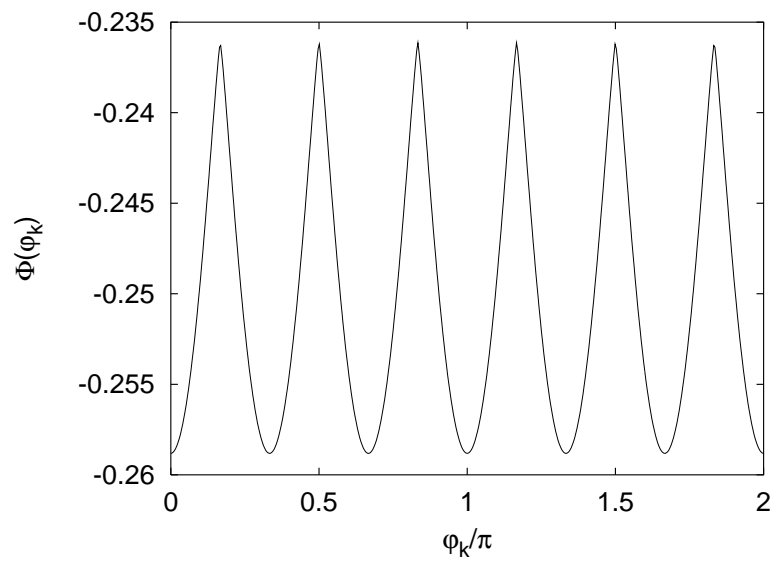

FIG. 9: Angular part $\Phi\left(\varphi_{k}\right)$ of the particle distribution function in Fourier-Laplace space in the limit $u / k \rightarrow 0$ for $\beta=0.28$ [see Eq. [16]).

considerably reduced in number. To arrive at the correct limit of the ordered honeycomb billiard, $t_{\text {dis }} \rightarrow \infty$ for $\delta r \rightarrow 0$. On the other hand, our description based on the step-time distribution (18) breaks down, when $t_{\text {dis }}$ approaches one, i.e, the time a particle needs to pass one channel. Diffusion in such strongly disordered channel billiards can be modeled by assuming that, on average, the particle's paths in different channels are uncorrelated, as we will explain below in detail.

The new step-time distribution (18) has a finite first and second moment. Therfore, the mean-square displacement in a disordered channel system is linear in time in the long-time limit, i.e., the particles behave diffusively. For smaller times, superdiffusion occurs depending on the value of $t_{d i s}$, as illustrated by the inset of Fig. [6 At a time of the order of $t_{d i s}$, a transition to the diffusive regime occurs. This is in aggreement with the results from our simulations. For decreasing disorder, the crossover time $t_{d i s}$ becomes larger and so does the time range where superdiffusion is found. Ultimately, in the limit $\delta r \rightarrow 0$ the time $t_{d i s}$ diverges and superdiffusion persists in the long-time limit.

At sufficiently strong disorder in the random channel billiards, the particle's paths in different channels are not correlated on average. Then we can consider a random walker that performs steps along the edges of the Voronoi tesselation. On average, the step length is equal to the edge length of the unperturbed honeycomb lattice, i.e., one in our scaling [27]. Furthermore, after each step the random walker changes its direction with the same probability $1 / 2$ either to the right or to the left by an angle that, on average, assumes a value of $\pi / 3$. This enables us to calculate the mean-square displacement:

$$
\left\langle\mathbf{r}^{2}\right\rangle=\left\langle\sum_{i, j=1}^{n} \mathbf{r}_{i} \cdot \mathbf{r}_{j}\right\rangle=n+2 \sum_{i=1}^{n} \sum_{j=1}^{i-1}\left\langle\mathbf{r}_{i} \cdot \mathbf{r}_{j}\right\rangle,
$$

where the vector $\mathbf{r}_{i}$ characterizes a single step with $\left|\mathbf{r}_{i}\right|=$ 
1. It is straightforward to show that $\left\langle\mathbf{r}_{i}\right\rangle=\mathbf{r}_{j} / 2^{i-j}$ for $i>j$ and we can set $\left\langle\mathbf{r}_{i} \cdot \mathbf{r}_{j}\right\rangle=\mathbf{r}_{j} \cdot \mathbf{r}_{j} / 2^{i-j}=1 / 2^{i-j}$. The mean-square displacement is then calculated using the formula for geometric sums:

$$
\left\langle\mathbf{r}^{2}\right\rangle=n+2 \sum_{i=1}^{n} \sum_{j=1}^{i-1} \frac{1}{2^{i-j}}=3 n-4+\frac{4}{2^{n}} .
$$

In the limit of $n \rightarrow \infty$ this becomes

$$
\left\langle\mathbf{r}^{2}\right\rangle=3 n
$$

To arrive at the mean-square displacement in terms of time $t$, we have to relate the number $n$ of steps to $t$. We first consider a particle path with a fixed angle $\alpha$ relative to the channel direction. For small channel widths $d$ so that details at the channel junctions can be neglected, the time $T(\alpha)$ to pass the channel is

$$
T(\alpha)=\frac{1}{\cos (\alpha)}
$$

The step number $n$ is then given by the average over all possible angles $\alpha$ :

$$
n=\frac{2}{\pi} \int_{0}^{\pi / 2} \frac{t}{T(\alpha)} d \alpha=\frac{2}{\pi} t
$$

With Eq. (21) and $\left\langle\mathbf{r}^{2}\right\rangle=4 D t$, we finally arrive at the diffusion constant

$$
D=\frac{3}{2 \pi}
$$

which is an excellent estimate for random channel billiards as illustrated by the perfect fit of the full line in Fig. 3 to the simulation results for $\delta r=0.3$ and $d=0.1$. Note that Eq. (24) does not depend on disorder. As soon as all long-time correlations are destroyed, any further increase of disorder does not affect the diffusion constant.

\section{CONCLUSIONS}

With the honeycomb billiard that can also be viewed as a system where hexagonal scatterers are placed on a triangular lattice, we have investigated a periodic extended billiard in detail. Though particles moving in the honeycomb billiard always have a finite horizon, there exist perfect paths where they move ballistically in one direction. We have clarified that almost perfect paths give rise to an overall superdiffusive behavior. Our simulations reveal a mean square displacement $\left\langle\mathbf{r}^{2}\right\rangle \propto t^{\nu}$ with a time exponent $\nu=1.72$. On the other hand, in our analytical treatment we have applied the Lévy walk model based on the formalism of continuous-time random walks by considering the long straight parts of almost perfect paths as effective steps. Assuming for their occurence a general distribution of the form $p(\epsilon) \propto \epsilon^{\beta}$, we can show that the mean square displacement possesses a time exponent $\nu=2-\beta$. A comparison with the simulation results then gives $\beta=0.28$ which means that almost perfect paths are less probable than other trajectories.

In contrast to previous treatments of Lévy walks, the directional distribution of steps in our model is not isotropic. Instead, steps are limited to the six possible channel directions, which are used by most of the perfect paths as suggested by our simulations. We therefore find that the time exponent of the mean square displacement corresponds to the one determined for one-dimensional systems [17, 18, 19]. Furthermore, our analysis reveals a starlike distribution of the particles' positions with a sixfold symmetry in accordance with our simulation results. So a limitation of the allowed step directions together with a step-time distribution that causes superdiffusion ultimately gives rise to an anisotropic particle distribution.

We have also introduced disorder into the honeycomb billiard so that the directions of the channels are randomized. In the limit of long times, these random channel billiards always display diffusive behavior. Transient superdiffusion is, however, visible in systems with weak disorder for small times. We explain it with the help of an exponential cut-off in the step-time distribution. For large disorder, correlations along the particle path between successive channels are lost on average. With the help of an elementary random walk model on a honeycomb lattice, we can estimate the diffusion constant which fits our simulation results very well.

Our investigation shows that the transport of particles in two-dimensional channel systems governed by the rule of specular reflection adds further insight to the current knowledge of extended billiards. Since we were motivated to the present study by light transport in foams, as mentioned in the introduction, the interesting question arises how the superdiffusion of classical particles in the honeycomb billiard will affect the analogous problem of interfering waves travelling along the channels. This question is also crucial for the relation between classical and quantum mechanics [28].

\section{Acknowledgments}

We would like to thank T. Franosch, T. Geisel, F. Jülicher, G. Maret, K. Richter, and H. Schanz for helpful discussions, and J. R. Shewchuk for making the program Triangle publicly available. H.S. acknowledges financial support from the Deutsche Forschungsgemeinschaft under Grant No. Sta 352/5-2. M.S. and H.S. thank the International Graduate College at the University of Konstanz for financial support. 


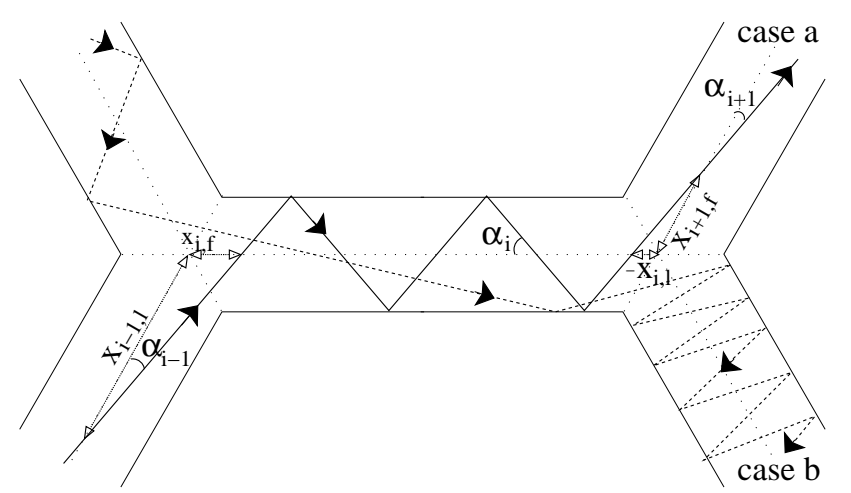

FIG. 10: The position of the particle within the $i$ th channel is given by the distance $\left|x_{i, f}\right|$ or $\left|x_{i, l}\right|$ between vertices of the underlying honeycomb structure (dotted lines) and the first or last intersection of the particle's path with the center line of the channel. The sign of $x_{i, l}$ and $x_{i, f}$ is negativ if the intersection is to the left of the vertices otherwise it is positive. The angle of the particle's path with the center line is denoted $\alpha_{i}$. It is always taken positive and does never change within a channel. For $\alpha_{i}<\pi / 6$, the particle proceeds into the channel $i+1$ situated either opposite (case a) or next (case b) to the last reflection in channel $i$.

\section{APPENDIX A: DISTRIBUTION OF EFFECTIVE STEP TIMES}

In this appendix we calculate the step-time distribution for our Lévy-walk model in Sec. IVA In concrete, we will consider paths with effective steps along the main lattice directions.

For special starting angles $\alpha_{1}$, so-called perfect paths exist where the particles run forever along one of the lattice axes, as illustrated in Fig. 2] A particle having started at an angle $\alpha_{1}+\epsilon$ will leave such a perfect path after an effective step time $t$. Analyzing special perfect paths to be defined below will allow us to calculate the step-time distribution $\lambda(t)$.

We first take a closer look at a general path of a particle crossing the $i$ th channel. The position and direction of the particle is characterized by $x_{i, f}$ (or $x_{i, l}$ ) and $\alpha_{i}$, as defined in Fig. [10] Depending on the position, a path with an angle $\alpha_{i}<\pi / 6$ proceeds into the new channel $i+1$ situated either opposite (case a in Fig. 10) or next (case b) to the last reflection in channel $i$. The new, respective angles therefore are $\alpha_{i+1}=\pi / 3-\alpha_{i}$ or $\alpha_{i+1}=$ $\pi / 3+\alpha_{i}$ (see Fig. 11). However, in channel $i+2$ one always finds $\alpha_{i+2}=\alpha_{i}$ since for $\alpha_{i+1}>\pi / 6$ only case a applies.

For reasons to become clear below, we now calculate the position $x_{i+2, f}$ in channel $i+2$ as a function of the parameters $\alpha_{i}$ and $x_{i, f}$ in the $i$ th channel assuming $\alpha_{i}<$ $\pi / 3$. We know already that this is fulfilled in every second channel. First, we calculate the position $x_{i, l}$ from the position $x_{i, f}$ :

$$
x_{i, l}=x_{i, f}+m_{i} d \cot \alpha_{i}-1,
$$

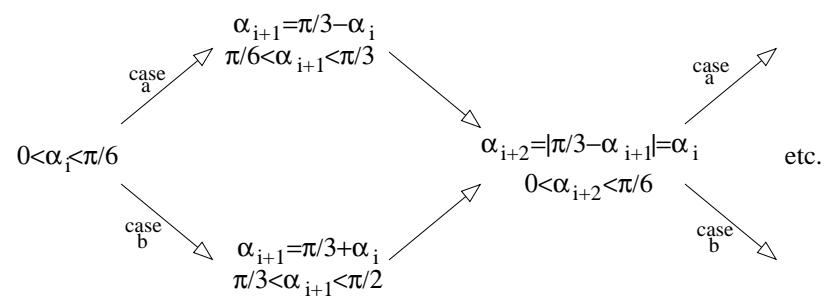

FIG. 11: All possible angles for a particle with a starting angle $\alpha_{i}<\pi / 6$. In every second channel the starting angle is repeated: $\alpha_{i+2 n}=\alpha_{i}$.

where the integer $m_{i}$ is the number of reflections in channel $i$. Secondly, with the law of sines we determine $x_{i+1, f}$ (see Fig. 10):

$$
x_{i+1, f}=\mp \frac{\sin \alpha_{i}}{\sin \alpha_{i+1}} x_{i, \text { last }} .
$$

The upper and lower sign belong, respectively, to case a or $\mathrm{b}$. The relations for channel $i+1$ equivalent to Eqs. (A1) and A2 are

$$
\begin{aligned}
& x_{i+1, l}=x_{i+1, f}+m_{i+1} d \cot \alpha_{i+1}-1, \\
& x_{i+2, f}=\mp \frac{\sin \alpha_{i+1}}{\sin \alpha_{i}} x_{i+1, l},
\end{aligned}
$$

where in Eq. (A4) $\alpha_{i+2}=\alpha_{i}$ was used. Finally, combining all equations (A1) to A4 , we obtain:

$$
\begin{aligned}
x_{i+2, f}= & x_{i, f}-1+m_{i} d \cot \alpha_{i} \\
& \pm \frac{\sin \left(\frac{\pi}{3} \mp \alpha_{i}\right)}{\sin \alpha_{i}} \mp \frac{\cos \left(\frac{\pi}{3} \mp \alpha_{i}\right)}{\sin \alpha_{i}} m_{i+1} d .
\end{aligned}
$$

From the multitude of possible perfect paths, we calculate the step-time distribution $\lambda(t)$ for a special class of perfect paths characterized by the requirement that the position $x_{i, f}$ in the channel is periodically repeated in every second channel, i. e., $x_{i+2 k, f}=x_{i, f}$ for all $k$. From Eq. (A5), this is the case for

$$
m_{i}=\frac{\tan \alpha_{i}}{d} \text { and } m_{i+1}=\frac{\tan \alpha_{i+1}}{d},
$$

where $\alpha_{i}$ now belongs to this perfect path. Examples are illustrated in Fig. 11 with paths (1) and (3).

For a particle with starting angle $\alpha_{i}+\epsilon$, not running on a perfect path, Eq. A5 applies as well with $\alpha_{i}$ replaced by $\alpha_{i}+\epsilon$. We can therefore calculate the difference in positions $\delta x_{i+2}=x_{i+2, f}^{\prime}-x_{i+2, f}$ in channel $i+2$ when the difference in channel $i$ is $\delta x_{i}=x_{i, f}^{\prime}-x_{i, f}$ :

$$
\delta x_{i+2}=\delta x_{i}+K_{\alpha_{i}} \frac{\sin \epsilon}{\sin \left(\alpha_{i}+\epsilon\right)}
$$

where

$$
K_{\alpha_{i}}=\frac{\mp 3-\sqrt{3} \tan \alpha_{i}}{ \pm \cos \alpha_{i}+\sqrt{3} \sin \alpha_{i}}
$$




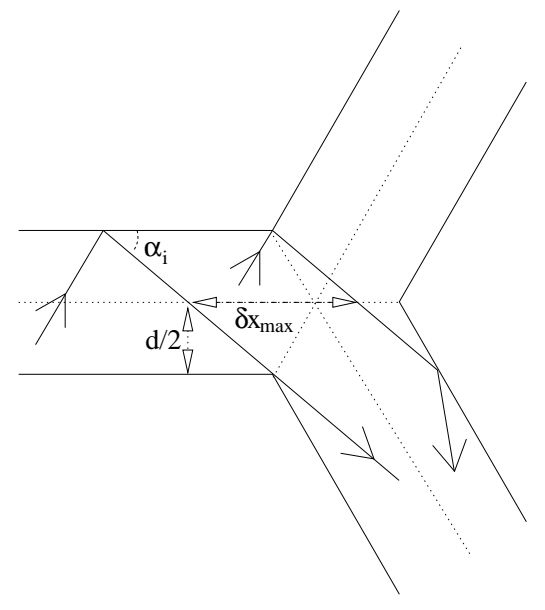

FIG. 12: Two particles can only enter the same channel if the distance between the last intersections of their paths with the channel center line is smaller than $\delta x_{\max }=d / \tan \alpha_{n}$.

and Eqs. A6 were used. We now assume that particles on the perfect path and its neighboring path start at the same position, i. e., $\delta x_{1}=0$. In the long-time limit the different behavior in channel $i$ and $i+1$ is irrelevant so that in the $n$th channel the difference in position $\delta x_{n}$ becomes

$$
\delta x_{n} \propto n \frac{\sin \epsilon}{\sin \left(\alpha_{1}+\epsilon\right)} .
$$

If $\delta x_{n}$ exceeds a threshold proportional to $\delta x_{\max }=$ $d / \tan \alpha_{1}$, particles travelling on the perfect path and its neighbor proceed into different channels (see Fig. 12). Therefore the maximum number of channels $n_{\max }$ where they travel through the same channels is

$n_{\max } \propto \delta x_{\max } \frac{\sin \left(\alpha_{1}+\epsilon\right)}{\sin \epsilon}=d\left(\sin \alpha_{1}+\frac{\tan \alpha_{1} \sin \alpha_{1}}{\tan \epsilon}\right)$.

Since the particles move ballistically and for small $\varepsilon$, the duration of an effective step therefore is

$$
t \propto n_{\max } \propto \frac{1}{\epsilon}
$$

The number of particles with starting angles in the intervall $\left[\alpha_{1}+\varepsilon, \alpha_{1}+\varepsilon+d \varepsilon\right]$ is $p(\varepsilon) d \varepsilon$, where $p(\varepsilon)$ is the distribution of starting angles close to a perfect path. Together with Eq. (A11), this determines the step-time distribution for $t \rightarrow \infty$ :

$$
\lambda(t) d t=p(\epsilon) d \epsilon \propto \frac{p(1 / t)}{t^{2}} d t .
$$

[1] M. Berry, Eur. J. Phys. 2, 91 (1981).

[2] A. J. Lichtenberg and M. A. Lieberman, Regular and Chaotic Dynamics, 2nd ed., Springer-Verlag, New York (1992).

[3] M. Berry in Semiclassical Mechanics of Regular and Irregular Motion, Les Houches, Session XXXVI, 1991, edited by G. Iooss, R. H. G. Helleman, and R. Stora, North-Holland, Amsterdam (1983).

[4] J. U. Nöckel and A. D. Stone, Nature 385, 45 (1997).

[5] D. Sweet, B. W. Zeff, E. Ott, and D. P. Lathrop, Physica D 154, 207 (2001).

[6] H. A. Lorentz, Proc. R. . Acad. Sci. Amsterdam 7, 438 (1905).

[7] L. A. Bunimovich and Ya. G. Sinai, Commun. Math. Phys. 78, 247 (1980); 78, 479 (1981).

[8] A. Zacherl, T. Geisel, J. Nierwetberg and G. Radons, Physics Letters 114A, 317 (1986).

[9] J. Machta and R. Zwanzig, Phys. Rev. Lett. 50, 1959 (1983).

[10] D. P. Sanders, Phys. Rev. E 71, 016220 (2005).

[11] D. P. Sanders and H. Larralde, e-Print archive; http://arXiv.org/cond-mat/0510654 (2005).

[12] D. Alonso, R. Artuso, G. Casati, and I. Guarneri, Phys. Rev. Lett. 82, 1859 (1999).

[13] B. Li, L. Wang, and B. Hu, Phys. Rev. Lett. 88, 223901 (2002).
[14] A. S. Gittings, R. Bandyopadhyay and D. J. Durian, Europhys. Lett. 65, 414 (2004).

[15] M. Schmiedeberg, MF Miri and H. Stark, Eur. Phys. J. E 18, 123 (2005).

[16] E. W. Montroll and G. H. Weiss, J. of Math. Phys. 6, 167 (1965).

[17] J. Klafter, A. Blumen, and M. F. Shlesinger, Phys. Rev. A 35, 3081 (1987).

[18] A. Blumen, G. Zumofen and J. Klafter, Phys. Rev. A 40, 3964 (1989).

[19] G. Zumofen and J. Klafter, Phys. Rev. E 47, 851 (1993).

[20] V. I. Arnol'd, Mathematical Methods of Classical Dynamics, Springer, New York (1978).

[21] P. J. Richens and M. V. Berry, Physica 2D, 495 (1981).

[22] M. Schmiedeberg and H. Stark, unpublished result.

[23] D.N. Armstead, B.R. Hunt and E. Ott, Phys. Rev. E 67, 021110 (2003).

[24] D. Weaire and N. Rivier, Contemp. Phys. 25, 59 (1984).

[25] A. Okabe, B. Boots, and K. Sugihara, Spatial Tessellations, Concepts and Applications of Voronoi Diagrams, John Wiley \& Sons, Chichester (2000).

[26] J. R. Shewchuk, http://www-2.cs.cmu.edu/ quake/triangle.html

[27] MF Miri and H. Stark, Europhys. Lett. 65, 567 (2004).

[28] T. Dittrich, B. Mehlig, H. Schanz, and U. Smilansky, Phys. Rev. E 57, 359 (1998). 